follow-up. Poor outcome predictors included female gender, chronic migraine (by ICHD-2 appendix criteria), medication overuse, major depression, anxiety disorders, school phobia, and OCD. Independent predictors for $\mathrm{CDH}$ persistence were medication overuse $(\mathrm{P}<0.05)$ and major depression $(\mathrm{P}<0.01)$. In contrast, migraine, age at $\mathrm{CDH}$ onset, and $\mathrm{CDH}$ duration were not significant predictors. (Wang S-J, Fuh J=L, Lu S-R, Juang K-D. Outcomes and predictors of chronic daily headache in adolescents: A 2-year longitudinal study. Neurology Feb 20 2007;68:591-596). (Reprints: Dr S-J Wang, Neurological Institute, Taipei Veterans General Hospital, Taipei, 112, Taiwan).

COMMENT. Whereas the incidence of $\mathrm{CDH}$ in adolescents declines over a 2-year follow-up, the relative prevalence of migraine diagnosis increases when compared to chronic tension-type headache, especially when the ICHD-2 appendix criteria are employed. In a previous study of headache at the $\mathrm{NIH}$ among adolescent girls, the prevalence was $29.1 \%$ in grades 6 through 10. Heavy alcohol consumption, high caffeine intake, and cigarette smoking were strongly linked to somatic complaints associated with headache, including stomachache, back pain, and morning fatigue (Ghandour RM et al. Arch Pediatr Adolesc Med 2004;158:797-803).

\title{
PSYCHOLOGICAL PROFILE OF CHILDREN WITH HEADACHE AND RECURRENT ABDOMINAL PAIN
}

The psychological profile of 70 patients (age range 4-18 years; mean 11 years) with headache, 70 with recurrent abdominal pain (RAP), and 70 controls was compared using the Child Behaviour Checklist 4-18 (CBCL). The CBCL profile was similar for headache and RAP and showed a statistically significant tendency toward problems in the Internalizing scale (anxiety, mood and somatic complaints) and no Externalizing (behavioral) symptoms cf controls. Patients with migraine compared to RAP showed a significant difference in the Attention Problems subscale but not in other subscales of the CBCL. The authors conclude that headache and RAP in children and adolescents have a similar etiology and require similar methods of treatment. (Galli F, D'Antuono G, Tarantino S et al. Headache and recurrent abdominal pain: a controlled study by the means of the Child Behaviour Checklist (CBCL). Cephalalgia March 2007;27:211-219 [abstract]).

COMMENT. The co-occurrence of headache and stomachache in young children constitutes a distinct syndrome with psychosocial implications requiring early intervention. (Borge AIH, Nordhagen R. Acta Paediatr 1995;84:795-802; Ped Neur Briefs Aug 1995). The co-occurrence group of patients in this longitudinal Norwegian study had emotional problems and low maternal emotional support, whereas school problems were not associated. In contrast, children with headache only were well-behaved, high achievers, and mothers were employed outside the home. Those with stomachache alone had an earlier onset of symptoms, they were well adapted emotionally, and mothers were less well educated. A causal link between recurrent abdominal pain and migraine is reported in 29 of 31 children with migraine found to have an underlying inflammatory lesion of the gastrointestinal tract (Mavromichalis I et al. Eur J Pediatr 1995;154:406-410). Symptoms of nausea, vomiting, and abdominal pain were associated with migraine headache in $93 \% .42 \%$, and $55 \%$ of patients, respectively. 